Article

\title{
Will Science and Consciousness Ever Meat? Complexity, Symmetry and Qualia
}

\section{Roger Vergauwen}

Center for Logic and Analytical Philosophy, Institute of Philosophy, Katholieke Universiteit Leuven, Kardinaal Mercierplein 2, 3000 Leuven, Belgium; E-Mail: roger.vergauwen@hiw.kuleuven.be

Received: 9 March 2010; in revised form: 25 May 2010 / Accepted: 23 June 2010 /

Published: 25 June 2010

\begin{abstract}
Within recent discussions in the Philosophy of Mind, the nature of conscious phenomenal states or qualia (also called 'raw feels' or the feel of 'what it is like to be') has been an important focus of interest. Proponents of Mind-Body Type-Identity theories have claimed that mental states can be reduced to neurophysiological states of the brain. Others have denied that such a reduction is possible; for them, there remains an explanatory gap. In this paper, functionalist, physicalist, epiphenomenalist, and biological models of the mind are discussed and compared. Donald Davidson's Anomalous Monism is proposed as a unifying framework for a non-reductive theory of qualia and consciousness. Downward Causation, Emergence through Symmetry-breaking, and Dynamical Systems Theory are used to show how consciousness and qualia emerge from their neural substrate and can also be causally efficacious.
\end{abstract}

Keywords: anomalous monism; consciousness; downward causation; dynamical systems; qualia; symmetry-breaking

\section{Introduction}

In his Philosophical Investigations, Ludwig Wittgenstein, while discussing the relation between consciousness and the brain, writes: "The feeling of an unbridgeable gulf between consciousness and brain-process: how does it come about that this does not come into the considerations of our ordinary life? This idea of a difference in kind is accompanied by slight giddiness, - which occurs when we are performing a piece of logical sleight-of-hand” [1]. Much of the discussion in contemporary Philosophy of Mind can be said to relate to this feeling of giddiness. More specifically, the nature of phenomenal states (also called 'raw feels', 'conscious sensations', or the feel of 'what it is like to be') and of 
consciousness in general have been an important focus of research. Qualia, then, are (first order) properties of mental states, e.g., 'seeing red', 'feeling pain', etc., and the concept is sometimes used more broadly used to indicate conscious experience in general. Explaining these qualia has been called 'the hard problem of consciousness': David Chalmers writes: "If any problem qualifies as the problem of consciousness, it is this one. In this central sense of 'consciousness', an organism is conscious if there is something it is like to be that organism, and a mental state is conscious if there is something it is like to be in that state. Sometimes terms such as 'phenomenal consciousness' and 'qualia' are also used here” [2]. Proponents of Mind-Body identity theories [3] claim that, in fact, there is no problem since it is perfectly possible to reduce these mental states and their properties to neurophysiological properties: "The Mind, Sir, is Meat.” Jack Copeland, in his book Artificial Intelligence: a Philosophical Introduction, quotes these words-uttered by Professor Ramsay, a character in the 1983 Colin Gregg movie after Virginia Woolf's novel To The Lighthouse-to give a succinct summary of the materialism or physicalism which is behind such theories. In the case of Functionalism, mental states are claimed to be reducible to functional or computational properties of the organism concerned. Others claim that a reduction is impossible and that, therefore, there remains an 'explanatory gap' [4]. So, while some consider consciousness and qualia as some kind of epiphenomena with no real existence in their own right, others want to say that there is a fact of the matter with respect to these phenomena and that therefore they should be given a separate ontological status and that we should also be philosophical realists about the things in question. The first group, let us call them reductionist, are antirealists in the sense that they believe that there is no fact of the matter as to the existence of the aforementioned phenomena or entities, unless maybe with respect to a certain background theory, but not as something existing in a mind-independent way [5].

During recent years, there also has grown an important body of research suggesting that mind and consciousness can be modeled along the lines of nonlinear complex dynamical systems, an approach that seeks to avoid certain metaphysical implications of the first two kinds of approaches and aims to show that cognitive activity and consciousness "are neither completely independent and different from brain activity nor simply identical, nor an epiphenomenon” [6].

This paper investigates the possibility of constructing an alternative to a (strongly) reductive theory of consciousness and qualia within the larger the framework of D. Chalmers' double-aspect theory of information approach to consciousness, which is based on the hypothesis that (at least some) information has two basic aspects, a physical aspect and a phenomenal or experiential (qualia) aspect [2]. The hypothesis tries to conceive of the relation between mental states and neural states in such a way that epiphenomenalism is avoided. Epiphenomenalism claims that mental events are caused by physical events, and that qualia are merely epiphenomenal in the sense that they cannot have any causal effect. Therefore, qualia are not 'real'. According to epiphenomenalism, if qualia are to be real, they have to be able to act as causal intermediaries for behavior, and if this is not the case, they have no role to play and are literally inexistent.

In the first part of this paper, the functionalist approach to mental states and qualia is discussed. On this view, a mental state is completely defined by its causal role in terms of the kinds of stimulation it is produced by and the kind of behavior it tends to produce, and by its interaction with other mental states. It will be argued that the (partial) reducibility of qualia in such a theory only incompletely accounts for their phenomenal or experiential aspect. 
Furthermore, if one wants to account for qualia as causally active, there is also the threat of epiphenomenalism, which is discussed in the next part. Epiphenomenalism implies the view that if mental states and qualia are physically realized by or supervene on physical properties, it is the 'physical realizers' who do the causal work, but the qualia themselves cannot be causally active nor can they in fact be functionally reducible. However that may be, epiphenomenalism also holds that there are law-like connections between mental and physical events. These laws are part of the reason why qualia are denied any causal powers since the connections account for the reducibility of mental states to physical states.

Davidson's anomalous monism, which is a token-identity theory of the mental, is discussed in a subsequent part. Anomalous monism holds that though there are no (strict or deterministic) general psychophysical laws (anomalism of the mental), any individual event described in mental terms is nevertheless also some physical event and can be so described, while at the same time, at least some mental events interact causally with physical events.

Anomalous monism allows one to avoid epiphenomenalism on the one hand, and on the other hand, it does not disallow the scientific study of consciousness. According to Davidson there are no general psychophysical laws. But this does not imply that no laws at all can be formulated here-especially not in the description of mental events at physical events.

It is here that the theory of nonlinear complex dynamical systems, which is discussed next, becomes relevant. This theory does not want to identify consciousness (and qualia) with neural states and nor does it want them to be completely independent of each other while explicitly also aiming at avoiding epiphenomenalism. The theory also emphasizes the embodiment of the mental and seems compatible with more biologically oriented theories of consciousness.

How in such a framework, which combines Chalmers' double-aspect-theory of information with Davidson anomalous monism and nonlinear complex dynamical systems, qualia and consciousness can emerge and be causally active is shown in a final part, where downward causation and symmetry-breaking in the embodiment of the mental [7] are suggested as possible mechanisms involved.

\section{Functionalism, Supervenience and Reduction}

In the philosophy of mind, functionalism is one of the theories used to clarify the mind-body problem. Functionalists hold that mental states, qualia, and cognitive states in general, can be viewed as the functional states of a machine or, rather, of a Turing-machine. In this view, the explanation of cognitive functions has to be done in terms of a causal-functional description of these functions. For example, a typical functional explanation of 'pain' would imply that pain is a functional state and as such, functions in the detection of tissue-damage. Of course, such a causal-functional state or role may cause the production of a certain behavior or of another functional state, which is the result of the first state. In functionalism, a mental state is defined in terms of three elements, which are causally related: the input-the stimulation, which causes the mental state, the causal interaction with other mental states, and the output, which is behavioral. In this sense, mental states are defined by means of a job description. Reductive explanations in science usually work this way. It is, e.g., known that organisms are able to transmit hereditary information. This was known even before the gene was discovered. Subsequent scientific investigations showed that in fact, DNA was responsible for this transmission and that 
therefore it did the job that was required. This kind of explanation of mental states is underpinned by the principle of mind-body supervenience, which is shared by many functionalists and non-functionalists [8].

\subsection{Principle of Mind-body Supervenience}

The mental supervenes on the physical in that any two things (objects, events, organisms, persons, etc.,) exactly alike in all physical properties cannot be different with respect to mental properties. That is, the lack of discernible physical properties entails the lack of discernible psychological properties. As it is sometimes put, there is 'no mental difference without a physical difference'.

The principle of supervenience is combined in functionalism with another principle, which is the principle of multiple realizability [70,71].

\subsection{Principle of Multiple Realizability}

Mental states are multiply realizable: any given mental state can be instantiated in (infinitely) many physical states.

For example, 'pain' as a mental state can be realized in all sorts of organisms, provided the organisms in question are pain-capable. What about the phenomenal state of pain, the quale 'pain', and, by extension, all qualia, including consciousness, since it makes perfectly good sense that there is a fact of the matter about what 'it is like to be conscious'? Here opinions diverge. Strong Physicalists, who want to reduce qualia to psychoneural identities, would hold that 'pain' is in fact nothing but 'C-fiber excitation' or some kind of other such identity. They claim that qualia are fully physically reducible. Frank Jackson famously constructed the 'Knowledge Argument' [9], and later withdrew it, against the reducibility of qualia and, indirectly, of consciousness. The story, roughly, goes like this: Mary is a scientist who for some reason or other has always been forced to investigate the world from within a closed room and from a black and white monitor in the room. She specializes in the neurophysiology of color vision and learns everything there is to be known about it. For example, she learns how colors are related to light-wavelengths, how these stimulate the retina, and how these stimulations are processed in the brain, thereby learning what these colors are. Now, the crux is the following: what will happen when Mary is released from her black and white room and is confronted with the 'real colors' outside? By hypothesis, she knows everything there is to be known about color vision. According to Jackson "it seems just obvious that she will learn something about the world and our visual experience of it. But then it is inescapable that her previous knowledge was incomplete. Ergo there is more to have than that, and Physicalism is false” [9]. This argument, together with Nagel's 'bat-argument' [10], is intended to show that qualia cannot be reduced. Let us note right away that the way this argument is presented by Jackson makes the argument about information and, more specifically, about physical information. Mary seems to have learned something new, the experience or phenomenal state of seeing colors, or 'what it is like to see colors'. An argument like this is considered to refute physicalism since it holds that ultimately, every fact about the human mind is a fact that can be described objectively or publicly. On the face of it, one might wonder how a fact about something that is informational can refute physicalism. Of course, we might understand the argument in such a way, as Jackson does, that, since Mary ex hypothesi knows 
everything that can be known physically about color vision, but that she doesn't know what 'it is like to see colors', and that moreover, the nature of any physical thing or property can be expressed in the vocabulary of physical science, the phenomenal nature of the experience ('what it is like to see colors', the quale) is not a physical property. But this nevertheless remains a proposition about informational states or knowledge-states. As we will see later, this fact will turn out to be crucial in the analysis of the non-reducibility of qualia and consciousness. However, this might also make us think that functionalism, which stresses the information-theoretic aspects of mental states, is a viable theory of qualia and offers also the advantage of reducibility-in-principle of consciousness. Sydney Shoemaker is a philosopher who works along these lines [11]. One of the arguments against qualia-reducibility is the thought experiment of 'inverted qualia' [8]. According to this experiment, it seems perfectly imaginable that people looking at things colored experience colors that are complementary. So it might be that things looking red to one person are experienced the way green things look to another person. At least, this seems conceivable without any contradiction. According to functionalism, this should be impossible, since it would mean that there could be beings that are functionally (psychologically) alike but who would differ in their qualitative states, in their experiences. Shoemaker argues that though individual qualia (such as the ones that characterize the individual experience of, e.g., pain or color) cannot be functionally defined, the concept of a quale is nevertheless a functional one. Here, he appeals to the notion of qualitative similarity, which he thinks "can be functionally defined in terms of its role in perceptual discrimination and recognition and in the fixation of perceptual beliefs... In terms of this we can define a notion of qualitative identity: and in terms of that we can explain what it is for a property to be a quale. Having this much of a functional account allows us to speak of qualia as being physically realized or implemented (in the sense in which any functional property can be physically realized or implemented)" [12]. However, such an explanation would seem to leave the phenomenal aspect of qualia unaccounted for [2].

Functionalism, then, is committed to the view that mental states and consciousness are defined in terms of causal roles with respect to behavioral and physical circumstances. Functionalism is surely committed to the view that systems that are alike in intrinsic physical properties must be alike with respect to their mental or psychological nature. However, almost unnoticed, in the attempt to define the mental in functional terms, another and even more important problem shows up, which is the problem of the causal structure and effectiveness of the mental. Functionalism defines mental states as causal states, but this has turned out to be their most problematic feature, especially for a theory that wants to account for the causal efficacy of qualia in a non-reductive framework.

\section{Epiphenomenalism and Mental Causation}

Causality and science go hand-in-hand. Entities may be said to exist qua entities only in as far as they have causal powers by means of which they can act and react: "being real and having causal powers go hand-in-hand; to deprive the mental of causal potency is in effect to deprive it of its reality” [8]. Jaegwon Kim has been instrumental in showing that especially qualia cannot be said to produce any causal effects and that they are therefore epiphenomena. Epiphenomenalism holds that while mental events are caused by physical events, mental events are merely epiphenomena, which are events without powers to cause anything. It follows that the mental qua mental does no causal work. The problem of mental causation 
comes down to the question of how the mind can have any causal powers in a world that is fundamentally physical. If mental phenomena, such as qualia and consciousness, are to be real, they somehow have to be able to act as causal intermediaries for behavior. If this is not the case, and if, therefore, their presence or absence does not make a causal difference, they can play no explanatory role and might as well not exist.

According to Kim, this is exactly what is the case with qualia. He claims that the "causal efficacy of mental properties is inconsistent with the acceptance of the following four claims: (i) physical closure (ii) causal exclusion (iii) mind-body supervenience and (iv) mental/physical property dualism-the view that mental properties are irreducible to physical properties” [13]. The first claim entails that the world is a physically closed domain, which means that if something has a cause, that cause cannot but be physical. The second claim implies that if something has a sufficient cause at a certain moment, then nothing else can be a sufficient cause for this very same thing (except in cases of overdetermination ). Briefly, this argument goes like this [14]: let us assume that the neural state P causes a mental state M. Suppose, furthermore, that $\mathrm{P}$ causes a further physical state or property $\mathrm{P}^{*}$, which causes in its turn the mental state $\mathrm{M}^{*}$, so that $\mathrm{M}$ and $\mathrm{M}^{*}$ supervene on $\mathrm{P}$ and $\mathrm{P}^{*}$ respectively. If $\mathrm{M}$ and $\mathrm{M}^{*}$ are supervenient mental properties then, by causal exclusion, if physical facts at a more basic level determine all the facts, then physical facts about physical processes must determine all causal facts, including all facts about the supposed causal role of the emergent properties $\mathrm{M}$ and $\mathrm{M}^{*}$, and therefore, again there is no causal work for the mental to do. Furthermore, appealing to substance-dualism, according to which there are two kinds of substances, physical ones and non-physical ones (e.g., the mind), each with their own specific non-reducible properties, is not very appealing either. It would seem that accepting this in an unqualified way may lead one to some kind of Cartesian dualism, or at the very least, it may look like a mystery. If mental phenomena are distinct from neural phenomena, it is unclear how these phenomena could exert any causal influence in the physical domain. Indeed, if mental phenomena supervene on physical properties, the correct way of analyzing them would be via their 'realizers', which are physical. But, then again, there is no special causal role for mental phenomena, and especially not for qualia. Kim, for one, concludes from this that mental states, in general, may be functionalizable. But he is adamant in claiming that individual qualia are not. In Kim's view, qualia do not fulfill any causal purpose and are even functionally irreducible as epiphenomena: "I am with those who believe that phenomenal properties of consciousness are not functional properties... I believe there are substantial and weighty reasons... to believe that qualia are functionally irreducible" [13]. Kim therefore comes close to philosophers, such as Daniel Dennett, who reject the belief in qualia as some kind of figment of a bad philosophy, and who are therefore usually called 'qualia-eliminativists' [15]. Kim's proposals in fact ignore the qualitative or phenomenal aspects of conscious states and disregard their causal relevance. There seem to be very good reasons to accept causal relevance with mental states and it will turn out that Davidson's anomalous monism may play a role here.

In order to see how this can be the case, let us first look at the general idea of causal efficacy for (epiphenomenal) mental states. Epiphenomenalism concedes that qualia, assuming they exist, are indeed distinct from, but nomologically correlated with, certain physical properties. However, according to Michael Pauen, it is logically possible that in this framework, since qualia are causally inert, there can be a disassociation of phenomenal and neural properties: "because a reductive explanation of phenomenal properties in, say, neuroscientific terms seems impossible in principle, there is no way to rule out that 
certain phenomenal events may occur in the absence of the corresponding neural events, and vice versa, even if our empirical investigations show strict and specific correlations between them” [16]. Such dissociations are exemplified in the inverted qualia thought experiment in which the qualitative states are interchanged even though the neural states are supposedly the same. However, this implies a causal difference in that it will have a causal effect on subsequent mental events and therefore this might also constitute a behavioral difference or a different brain activity. Moreover, our first-person knowledge of phenomenal states will be endangered if it is assumed that mental properties and states are causally impotent. The reason for this is that the justification of psycho-physical laws needs first-person justification and phenomenal knowledge, since, if qualia epiphenomenalism is true, no experimental evidence can detect psychophysical dissociations [16]. Phenomenal knowledge is also necessary to explain the explanatory gap argument mentioned before [4] for the simple reason that such an explanation would be impossible if we would have no knowledge of phenomenal states. Therefore, if phenomenal states would be causally inert, but nomologically connected to certain neural properties, "qualia-epiphenomenalism cannot appeal to experimental evidence in order to establish psychophysical laws because scientific experiments would not need the disconfirmation constraint, if qualia-epiphenomenalism were true... If we want to avoid these difficulties, then we should subscribe to an account that takes phenomenal properties as causally relevant by themselves, such that relevant differences on the phenomenal level have corresponding differences on the causal level: feeling causes” [16]. The existence of psychophysical laws remains, however, a necessity. Psychophysical laws warrant scientific access to the mental in general and qualia in particular. In fact, as it will turn out, the causal efficacy of mental states is also necessary to solve the aforementioned problem of the explanatory gap. If phenomenal states (qualia, or first-person-knowledge) are identified with neural states, then the mental state is identical to the neural state. The problem is that such an identification, if at all possible, would not account for the phenomenal character of these mental states. It would not explain why "pain" feels like pain or "red" looks like red. Pauen thinks that a precise description of the relevant phenomenal states would be one of the prerequisites of a solution to the problem, which could be solved if we could capture phenomenal properties in terms of their causes and effects. Pauen also thinks that it cannot be ruled out that this can be done sooner or later by scientific research [17]. In our view, no progress can be made in this respect unless we further clarify what the nature could be of the laws that connect the physical and mental. And it is here that Donald Davidson's anomalous monism may be a good starting point.

\section{Anomalous Monism and the Mental}

Donald Davidson's central idea in the philosophy of mind is that of anomalous monism. It implies a token-identity theory of the relation between mind and body. In such theories, mental kinds (or mental states) can be identified with physical states. So e.g., "Pain-instances” are not only instances of mental states, but also some kinds of physical states, so that mental kinds fall under certain physical kinds (one of the classical examples here is that pain is "C-fiber activation"). But according to token-identity theories, there may be several physical kinds under which a mental kind falls. In this sense, token-identity theories of the relation between mind and body differ from type-identity theories, which claim that mental kinds themselves are physical kinds and that therefore, token-identity theories are weaker than type-identity theories. 
So, in both cases, there is an in-principle reducibility of the mental to the physical. However, token-identity asserts that: "while one cannot identify mental states with physical states in the sense that for any X., X. is M if and only if X. is in P., one can for any instance of being in a mental state, find a physical state such that being in it at that time is identical to being in the mental state” [18]. Davidson's anomalous monism rests on three principles which can be summarized as follows [19]:

(a) Causal interaction: at least some mental events interact causally with physical events.

(b) Nomological character of causality: events that are related as cause and effect fall under strict deterministic laws.

(c) Anomalism of the mental: there are no strict deterministic laws on the basis of which mental events can be explained and predicted.

Before briefly discussing these principles, let us point out what we consider to be a misinterpretation of Davidson. Kim, who has been a long-time critic of Davidson's approach, seems not to notice that Davidson is not advocating a kind of "lawlessness of the mental”. Kim seems to misconstrue Davidson's proposal in this direction: "one such assumption centers on the question whether there are laws connecting mental phenomena with physical phenomena that are needed to underwrite causal connections between them. Davidson's celebrated anomalism of the mental states that there can be no such law, whence it follows that there can be no causal relations between mental and physical phenomena. Davidson however is a believer in mental causation... Thus the challenge posed by Davidson's psychophysical anomalism is to answer the following question: how can anomalous mental properties that are not fit for laws, be causally efficacious properties?” [8]. However, Davidson merely claims that there are no "strict deterministic laws" that could function as "bridge laws" between the mental and the physical. On the face of it, this is not the denial of any laws as Kim implies [19]. Maybe non-deterministic or other kinds of laws are possible and one wonders to what extent Kim is setting up a straw man here. Of course, Davidson believes in the causal efficacy of the mental; this belief needs justification.

The first principle, the principle of causal interaction of the mental, is taken more or less for granted. Davidson mentions as an example how in the case of an event like the sinking of the Bismarck, which is a physical action, various mental events such as beliefs, calculations, intentional actions, and changes of belief have played a causal role and he concludes that "I think reasons could be given for the view that all mental events, perhaps through causal relations with other mental events, have causal intercourse with physical event. But if there are mental events that have no physical events as causes or effects, the argument will not touch them" [19]. Davidson thus avoids epiphenomenalism. It is clear that Kim could not accept this, but, as we will show later, Davidson is indeed on the right track, at least in principle. Davidson's theory is, furthermore, monistic, because it is inconsistent with Descartes' claim that mental and physical events may not be identical, and in fact, are properties of different kinds of substances between which there is an interaction.

By appealing to the concept of a strict law, Davidson wants to say that such laws dispense with causal concepts and also with so-called ceteris paribus clauses. An idealized or strict science considers the world as causally closed [20] and therefore has no need for ceteris paribus clauses. This is why only physics can aspire to the state this strict of a science; it has only strict laws and no ceteris paribus clauses [21]. From this, it follows immediately that many of the traditional sciences, such as psychology, 
evolutionary theory, and others, in fact, are also anomalous: "much of what I said about what distinguishes mental concepts from the concepts of a developed science could also be said to [distinguish] the concepts of many of the special sciences such as biology, geology, and meteorology" [22]. The reason for this is that in such sciences, a neat translation of the concepts used there into the language of physics is not possible, and therefore these sciences can all be said to be anomalous. However, nobody doubts that there are nevertheless laws in these sciences. So, again, when Davidson makes a plea for the anomalism of the mental he is not, in our view, claiming that there are no laws whatsoever, but only that these laws do not line up with the strict laws of physics (at least the kind of physics that he has in mind). What this discussion of Davidson's anomalous monism shows is that the idea of the epiphenomenalism of the mental and the supposed lack of causal powers of mental states and qualia may be due to a one-sided idea of the relation between the mental and physical, a further discussion of which may help us to reveal something of the true nature of the mental.

\section{Enter Nonlinear Complex Dynamical Systems}

The study of nonlinear complex dynamical systems has become an important field of study over the last few decades [6]. With respect to its relevance to the philosophy of mind, this approach explicitly wants to present a position which is non-reductionist: "some philosophers have criticized that a physical description of brain states fails to grasp the essence of what is a phenomenal state. Proponents of the opposite view have argued that notions of phenomenal states can be reduced to notions of neurophysiological states of the brain ... both positions are ideological reductionisms and exaggerations which are neither justified by research nor very helpful in research ... according to the complex systems approach, neurophysiological states and mental states are modeled by mathematical formalisms without reductionist ambitions" [6]. This is why dynamical modeling puts a lot of weight on finding mathematical rules and equations which describe the phenomena in question and their evolution [23]. What is important here is the mathematical modeling of the biophysical processes in the brain.

This approach heavily stresses the idea of the embodiment of the mind and therefore, in our view, goes very well with an approach to consciousness, such as the one proposed by Gerald Edelman [24]. He begins by stressing that though it is sometimes claimed that any attempt to reduce phenomenal or subjective experience (qualia) to neural activities leads to an explanatory gap, which constitutes the hard problem of consciousness [2,4], this need not be a problem because in a certain sense, a reduction is possible: "the neural systems underlying consciousness arose to enable high-order discriminations in a multidimensional space of signals [and] qualia are those discriminations. Differences in qualia correlate with differences in the neural structure and the dynamics that underlie them and I have stressed that it is the distinctions among the entire set of experienced qualia that allow the specific defining property of each quale to appear" [24]. However, a scientific account of the individual experiences of these qualia is not to be expected since: "a scientific theory cannot presume to replicate the experience that it describes or explains: a theory to account for a hurricane is not a hurricane. A third-person description by a theorist of the qualia associated with wine tasting can, for example, take detailed account of the reported personal experiences of that theorist and his human subjects. It cannot, however directly convey or induce qualia by description: to experience the discriminations of an individual it is necessary to be that individual” [24]. In other words, simulation is not duplication. So Edelman, who has a rather broad 
conception of qualia, and in fact states that "consciousness consists of qualia, by which I mean ... the entire rich panoply of subjective experience" [24], claims that qualia are reducible, but only with respect to their differences (and similarities). What he has in mind comes close to a functionalist approach in which not the qualia themselves, but their differences and similarities can be functionalized, a view that is also held by Shoemaker and Kim: "Intrinsic qualities of qualia are not functionalizable and therefore are irreducible, and hence causally impotent....certain important relational facts about qualia, in particular their similarities and dissimilarities, are detectable and functionalizable, and can enjoy causal powers as full members of the physical world” [13].

We have already indicated earlier that this kind of functionalization may not be sufficient and that qualia in themselves could be causally active. A first indication of how this could be can already be found in the theory of Neuronal Group Selection, a neurobiological model proposed by Edelman himself, where he uses the concept of a Dynamic Core [25]. Here a distinction is made between primary consciousness and higher-order consciousness [14]. Primary consciousness involves the ability to construct "mental scenes" with limited informational or symbolic content, such as may be found in an animal's ability to learn and adapt its behavior, and which is more or less immediate. Higher-order consciousness evolves later and is related to semantic capabilities, as is found in its fully developed form in human beings who have a fully fledged language with syntax and semantics. It therefore goes beyond primary consciousness as a "remembered present" but extends into the past and the future. Essential properties of this kind of consciousness are unity, informativeness, and privateness (accessible only to conscious agents).

The theory further provides a neurophysiological description of the likely mechanism at the basis of conscious awareness. It is argued that a conscious state is a dynamical process within the cerebral cortex in which functional interconnectedness is created by rapid, two-way (re-entrant), neural interactions. Such a functional integration is called a "dynamic core". This core is itself, then, a group of neurons-a neural state-and it is the sets of neurons involved in the (functional) relations between them that constitute the nature and content of consciousness at any given moment. Such dynamic core(s) may be sensitive to further bodily (sensory) inputs or from other brain systems with which they interact, as well as with itself. This gives rise to extremely complicated patterns of interactions, but what is also important is that the whole system is embodied, especially with respect to the subjectivity of experience. The activity of the dynamic core leads to successive discriminatory states, which entail sets of phenomenal experiences: "but who or what experiences these higher-order discriminations, these qualia? A reasonable suggestion emerges from the facts of embodiment. The brain and body exchange signals from the earliest times of neuronal development and together they interact with the world" [24]. Consciousness in this view arises from re-entrant interactions among neural populations and the causal activity is produced by the dynamic core; so, the qualia emerging from this core are caused by it, but, according to Edelman, this does not necessarily lead to epiphenomenalism because these emerging qualia are informational structures even if they are not causal and it may sometimes be useful to talk about these qualitative informational states "as-if” they are causal, depending on the level of description. This does not, however, imply epiphenomenalism, since "There is, however, no need to conclude that $\mathrm{C}$ [qualia] is therefore meaningless and unnecessary. $\mathrm{C}$ states [qualia-states] are informational even if not causal. C states are the discriminations entailed by causal transactions among C' [neural states]” [24]. The qualia, then, are the reflections of the permanent causal interactions in the (complex) dynamic core, 
but are themselves not causally active: "underlying each quale are distinct neuroanatomical structures and neural dynamics that together account for the specific and distinctive phenomenal property of that quale" [24]. As for the phenomenal experience of the quale itself, the theory holds that it is no problem, and that therefore, the "hard problem "and the problem of the "explanatory gap" [2,4] are ill posed, because the need for a phenotype experiencing and giving rise to the qualia is no hindrance for a scientific theory of consciousness and, moreover, "if the phenomenal part of conscious experience that constitutes its entailed distinctions is irreducible, so is the fact that physics has not explained why there is something rather than nothing” [24].

Edelman recognizes a phenomenal part of experience as real and irreducible, thus rejecting epiphenomenalism, but he gets into an awkward position with respect to functionalism. On the one hand, with his biological theory of neural group selection and embodiment, Edelman wants to explicitly do away with information processing, functionalist, models of the brain [26]. But on the other hand, he comes up with the idea of qualia as being informational states that reflect the discriminations entailed by 'real' causal interactions in the dynamic core and that qualia are neither useless nor unnecessary [24]-an idea that can be called functionalist. At the same time, he denies these qualia any causal efficacy as a "regular" functionalist would do. Still, in Edelman's view, qualia are real. But from a functionalist perspective, causal efficacy is a criterion for existence. After all, consciousness does seem to make a difference, but it is not clear if consciousness can be causally active and neither is it immediately clear how all of this can be reconciled with Davidson's anomalous monism, which was discussed earlier and which explicitly endows mental states with causal efficacy. In order to see how this is possible, a closer look at how exactly qualia might emerge from their neural substrates and how this process can be described is useful. In this respect, Edelman writes: "underlying each quale are distinct neuroanatomical structures and neural dynamics that together account for the specific and distinctive property of the quale. Qualia then reflect the causal sequences of the underlying metastable neural states of the complex dynamic core” [24]. Indeed, Edelman rightly stresses the neural dynamics that, together with neuroanatomical structures, account for the qualia.

These dynamics, and especially the dynamic core hypothesis, are very well compatible with the idea that the brain is some kind of complex dynamical system, or that the brain can be described as such [14]. It is therefore natural that the theory of nonlinear complex dynamical systems should be a useful tool for the study of the phenomena under discussion [27]. In several publications, Klaus Mainzer has developed the importance of this approach and shown its relevance for many different disciplines, such as economics, culture and society, brain research, and the philosophy of mind. In this view, "the brain is considered as a complex dynamical system of firing and non-firing neurons, self-organizing in macroscopic patterns of cell assemblies by neurochemical interactions. Their dynamical attractors are correlated with states of perception, emotion, thoughts, or even consciousness” [28]. This approach is characterized by non-linearity, in which "the dynamics are modeled by a non-linear equation where causes and effects are no longer proportional. Feedback loops and complex interactions of system elements can lead to the self-organization (emergence) of new structures, but also to chaos” [29]. Very explicitly, and with respect to the philosophy of mind, this theory seeks to avoid the Scylla of dualism and the Charybdis of reductionism. The theory both considers to be one-sided and outdated positions. The emergence of mental states is described by Mainzer as follows: "The emergence of mental states ... is explained by the evolution of (macroscopic) order parameters of cerebral assemblies which are caused 
by nonlinear (microscopic) interactions of neural cells in learning strategies far from thermal equilibrium. Cell assemblies with mental states are interpreted as attractors ... of phase transitions” [6]. These parameters cannot be identified with the states of single cerebral cells, and therefore "emergent effects of the whole system are system effects which cannot be reduced to the single elements. Philosophically, the whole is more than the sum of its parts” [6].

According to Mainzer, the complex system approach is able to model the neural interactions at the lower level and consequently the emergence of macroscopic cognitive structures. This modeling heavily relies on mathematical formalisms without any implication of reductionism [6]. The mathematical principles governing nonlinear complex dynamical systems do not depend on the particular setup of the human brain and it can therefore be expected that it would in principle be possible to create artificial minds with their own kinds of cognitive states [29]. The human brain is a natural model of the aforementioned principles but, pace Searle [30], other “artificial”, i.e., non-biological, implementations are possible. The emergence of qualia finds a natural explanation in this theory when one considers embodiment and bodily interaction with the environment: "from a neurophysiological point of view the old problem of 'qualia' is also solvable. Qualia are properties that are consciously experienced by a person ... qualia in this sense emerge through the interaction of self-conscious organisms bodily with their environment, which can be explained via nonlinear dynamics of complex systems ... Therefore, we can explain the dynamics of subjective feelings and experiences, but, of course, the actual feeling is an individual experience" [6]. Some have even hypothesized that within such a framework, the concept of "symmetry-breaking" may be closely related to the emergence of qualia: "the different phases in the case of living brain tissue are the various qualia. Just as liquid, solid, and gas are phases of water, so are touch, sound, and odor different phases of brain systems. Dynamical rearrangement of symmetry gives the kinds of qualia whereas symmetry breaking within phase gives the values for a quale” [7]. Furthermore, certain properties that qualia are commonly said to have, such as "uniqueness", could be accounted for within this framework: "if at least certain properties of the mind can be seen as nonlinear dynamical systems that have a chaotic and unpredictable nature, then the uniqueness of qualitative experience seems to be a genuine possibility" [27].

We will come back to this in the next part, but let us pause for a moment and take stock. Qualia and mental states require causal efficacy for their existence. Davidson's anomalous monism assumes that mental states are causal. A discussion of Edelman's neurobiological model has shown that at first sight, though qualia are real as informational states, in this approach they seem to be doomed to causal impotency. The theory of nonlinear complex dynamical systems can provide a framework for the emergence of qualia and at the same time give support to Edelman's approach, but how and in what sense it can account for the causal powers of mental states and qualia is not immediately clear. Finally, Davidson's anomalous monism claims that there are no strict deterministic laws on the basis of which mental events can be predicted and explain. Is there, therefore, a way of describing and explaining qualia and consciousness such that Levine's 'explanatory gap' and Chalmers' 'hard problem” can be made sense of without having to say, as Edelman does, that the problem is ill-posed and is merely "framed in the expectation that, for an observer, a theoretical construct can lead by description to the experiencing of the phenomenal quality being described" [24]? 


\section{Are We Forever at a Loss? Downward Causation and Third-Order Emergence}

According to Chalmers, the search for the solution to the hard problem of consciousness, thereby bridging the explanatory gap, is characterized by the lack of an account of "why" and "how". Since we cannot explain consciousness on the cheap, it is sometimes suggested that we need an 'extra ingredient' in the explanation. Chalmers considers several of these "extra ingredients", all of which he finds lacking the necessary explanatory power when it comes to an explanation of the phenomenal side of conscious experience. He explicitly mentions non-linear dynamical and chaotic systems, which he considers together with the proponents of quantum mechanical theories and non-algorithmic processing, such as Roger Penrose's theory [31]. He is rather pessimistic about these extra ingredients. So, e.g., with respect to nonlinear dynamical systems he writes: "unfortunately, these suggestions all suffer from the same old problems. The same goes for nonlinear and chaotic dynamics. These might provide a novel account of the dynamics of cognitive functioning, quite different from that given by standard methods in cognitive science. But from dynamics, one only gets more dynamics. The question about experience remains as mysterious as ever" [2]. The same would hold with novel discoveries in neurophysiology, as would be the case with Edelman's proposals. Such discoveries might well further elucidate the nature of cognitive functions, but it is not as if they, as Chalmers writes, "will suddenly discover a phenomenal glow inside a neuron!"

At this point, one may do any of two things. Either one holds that "the buck stops here," in the sense that, as Edelman holds, qualia are real and are not just epiphenomenal, but that they are a-causal. But then, of course, what is the status of these qualia as entities? One might as well say that they do not exist then. Or one may attempt to construct a (mathematical) model of the processes that give rise to conscious experience and qualia, but then again, one may risk a kind of explanatory gap and have no real account of their causal powers. There is, however a third option, which will allow us to draw the threads together, and which allows a unification of the proposals discussed before. Or, if one wants a fourth one, an option we will not take here, there is the denial of the possibility of any theory of consciousness at all, because it is just not possible for human beings to construct such a theory [32], thus declaring the scientific study of consciousness as off-limits. The third option starts from the consideration that Chalmers is much too pessimistic with respect to what he calls the extra ingredients that have been proposed in the explanation of consciousness. Indeed, such an option, which concentrates on the notions of embodiment and information, could start from a possible solution proposed by Chalmers, but not only from his solution. According to Chalmers, a (non-reductive) theory of consciousness will have to take into account several principles connecting the properties of physical processes to the properties of experience [2]. Among the several principles he mentions is the "double-aspect theory" of information, which he considers a basic principle. The hypothesis, then, is that information has two fundamental aspects, a physical one and an experiential or phenomenal one, and that, furthermore, phenomenal consciousness, by virtue of its status, is one aspect of information where the other aspect is found embodied in physical processing [2]. Physical information is, as embodied information, then, "a difference that makes a difference". Chalmers concludes that information is therefore a natural candidate to play a role in a fundamental theory of consciousness. However, it is not clear how to account for the causal nature of consciousness and of qualia in such a theory so that epiphenomenalism might still be an option. The informational nature of phenomenal consciousness and qualia, which we find with Chalmers, is also a property of 
Edelman's approach and compatible with the theory of nonlinear complex dynamical systems and is, therefore, also a good starting point for such an account.

Edelman, again, notices that qualia emerge from their physical substrate. He observes that as informational structures, qualia reflect the causal transactions between the successive stages in the dynamic core. They constitute a "phenomenal transform”. While in themselves, they are not causally active, they are nevertheless entities in their own right. If we want to make sense of the causal nature of qualia and phenomenal consciousness, it is important, firstly, to notice that qualia emerge from their neural substrates. They are, therefore, a direct consequence of the embodiment of the mental, and at the same time, the expression of the dual nature of physically embodied information. The concept of emergence has a rich history [14] and it comes in several variations. The concept was especially rife in the philosophy of biology throughout the 20th century, where it was proposed as an alternative to vitalist accounts of the origins of life. Gradually, the concept came to be applied to the mind-brain problem and especially the emergence of consciousness.

The concept itself is by no means easy to define, but there are some useful characteristics that it may be said to have: according to Kim [8], emergentism implies that firstly, all that exists in the space-time world are the basic particles recognized in physics and their aggregates. It further entails that when aggregates and their articles attain an appropriate level of structural complexity, genuinely new (higher-order) properties emerge to characterize these structured systems and that these emergent properties are irreducible to, and unpredictable from, the lower-level phenomena from which they emerge. Finally, once these higher-level properties (e.g., mental states or qualia) emerge, they are able to manifest causal powers in their own right, thus affecting the lower-level phenomena ("downward causation"). This idea of "the whole being more than its parts" is explicitly present in the non-linear dynamical systems approach and the concept of downward causation is used extensively nowadays in psychology, cognitive sciences, and the interpretation of biologically complex data [14]. To a reductionist philosopher, downward causation, which we would suggest is a property of mental states and qualia, is outright unacceptable, because it would mean that one gives up the causal closure of the physical world, thereby invoking weird entities that supposedly would have causal powers, while remaining irreducible themselves: "to give up this principle [causal closure] is to acknowledge that there can in principle be no complete physical theory of physical phenomena, that theoretical physics, in so far as it aspires to be a complete theory, must cease to be pure physics and invoke irreducibly non-physical causal powers-vital principles, entelechies, psychic energies, élan vital, or whatnot” [33]. However, not only is the concept of a complete physical theory in itself vague and maybe even self-contradictory, but it is also possible to make sense of downward causation without appealing to the weird causal powers mentioned by Kim. The concept that is needed here is that of causation as "constraint" [34] or "selection" [35]. In physical systems, the causal powers of the objects are not determined only by the physical properties of the constituents and the laws of physics, but also by the organizational patterns. These patterns can have "downward causal capacities" in that they can affect which causal powers of the composing constituents are activated. The whole is, therefore, not the sum of its parts, and neither is it any simple function of it. "Higher-order patterns can have a degree of independence from their underlying physical realizations and can exert what might be called downward causal influences without requiring any objectionable form of emergentism by which higher-order properties would alter the underlying laws of physics. Higher-order properties act by the selective activation of physical powers, 
not by their alteration" [35]. Therefore, Van Gulick doubts the claim of dependency of the mental on the physical and speculates that such higher-order patterns constitute an order that manifests itself in our world via physical realizations. This order is informational first and foremost and can, in our view, at least in part be said to constitute the phenomenal aspect of physically embodied information: "there seems to be no way around the idea that form, or organization, can be a legitimate locus of emergent causal powers... namely that once a macro level structure has emerged, it is capable of acting as an organized whole whose activities constrain (that is, dynamically constrain) the unfolding of causal processes at lower levels of organization in ways that were not possible prior to emergence and so, in part, in virtue of the emergent organization” [36]. Juarrero takes a similar stance. Starting from a distinction between context-free and context-sensitive constraints, she claims that nonlinear systems impose contextual constraints on data components. The history of the system here is essential to its workings in that "the higher level's self-organization is the change in probability of the lower-level events. Top-down causes cause by changing the prior probability of the components' behavior, which they do as second-order contextual constraints” [34]. Due to the emergence of relatively autonomous levels of organization, new relatively autonomous qualities also arise with new modes of being, which can carry with them new modes of causality [34]. Qualia and consciousness, considered as informational structures, might now be said to constitute a level of higher-order organization, which can subsequently, by downward causation, have further effects on the whole of the system. The ability of Chalmers' double-aspect theory and nonlinear dynamical systems theory to agree here this shows that, pace Edelman, qualia can be causally active because of their informational aspect, an aspect which is explicitly endorsed by Edelman. The agreement also shows that qualia are not epiphenomenal and neither does this agreement contradict Davidson's token-identity theory of the mental. Earlier, we have noted that though Davidson's anomalous monism holds that there are no strict deterministic laws on the basis of which mental events can be predicted and explained, this does not preclude the possibility of there being laws that could account for mental events. In all likelihood, if any laws exist, on the basis of which mental events (consciousness and qualia) can be explained or predicted, these laws might be deterministic but non-linear and chaotic or, alternatively, stochastic and nonlinear, which implies that the emergence of patterns here could only be explained probabilistically [29,35]. Our approach has attempted to show just how this causal efficacy may be explained as downward causation, but it still requires an explanation of the mechanism of how emergence happens.

Terence Deacon has recently [37] offered a novel account of emergence, which sheds light on this. In general, the emergentist's aim might be said to show how, within complex systems, new entities emerge that exhibit novel causal powers. Deacon starts from the assumption, already present with Van Gulick and Juarrero, that in emergent phenomena, the physical laws governing the constituents of a system should not be violated and that an additional account should be given of the regularities (with respect to configuration) affecting constituent interactions. Furthermore, the relative autonomy and causal efficacy of such "holistic" emergent phenomena should be accounted for. Instead of making a commonly held distinction between "strong" and "weak" emergence [38], Deacon makes a threefold distinction between different kinds of emergent systems. In doing so, three interconnected hierarchical levels of emergence can be described. The first kind of emergence is "first-order emergence" or "supervenient emergence". This happens in systems in which relational properties determine the emergent higher-order properties, For example, the liquidity of water is determined by the aggregation of water molecules [37]. A second 
kind of emergence, "second-order emergence”, is present in diachronic symmetry-breaking. This kind is typically also found in living systems and mental processes: "in contrast, there is a self-differentiating feature to living and mental processes, which retains and undermines aspects of self-similarity. This characteristic breakdown of self-similarity or symmetry-breaking is now recognized in numerous kinds of complex phenomena, including systems far simpler than living systems. These complex emergent phenomena share this characteristic change of ensemble properties across time, and are often computationally unpredictable” [37]. It should come as no surprise that the concept of symmetry [39] and symmetry-breaking is mentioned here with respect to (second-order) emergence. We have already noted that symmetry-breaking might be an important element in the explanation of qualia [7]. To begin with, there is every reason to believe that the very concept of information is intimately related to symmetry-breaking.

According to John Collier [40], the process of symmetry breaking constitutes the very origin of information and it can be seen at work throughout cosmology and in the early universe, but it can also be applied to the acquisition and construction of information in perception and in many cognitive processes [40]. Moreover, symmetry-breaking and its description in the mathematical formalism of nonlinear dynamics can also be applied to biological and sociological models [39]. The process of symmetry-breaking was mentioned earlier as the source of the emergence of qualia within the brain, and therefore as an example of embodiment of the mental [7]. However, it was suggested that somehow, symmetry-breaking (within phase) and the dynamical rearrangement of symmetry were identical to the qualia. It appears now that symmetry-breaking may be at the origin of the emergence of qualia as causally active entities, but it is not identical to them. In view of the foregoing, an account of qualia might, therefore, also consider their informational dimension. It is here that third-older emergence may play a role. Third-order emergence involves, in addition, information or memory. It is the kind of higher-order regularity which can additionally exert a cumulative influence over the entire causal future of the system, thus encompassing the evolution of second-order processes. According to Deacon, this involves an additional leap of recursive causality: "the relationship implicit in third-order phenomena demands a combination of multi-scale, historical, and semiotic analyses. Thus, living and cognitive processes require introducing concepts such as representation, information, and function in order to capture the logic of the most salient emergent phenomena” [37]. The level of third-order emergence, therefore, is "the point where physical causality constitutes significance" [14]. Among these third-order emergent properties, Deacon explicitly considers processes such as 'mental experiences' [37], suggesting that the kind of causation involved here is downward causation. Interestingly, he also claims, in opposition to the strong functionalists, that mental processes cannot be instantiated by determinate computational machine operations. But he does not deny that third-order emergent processes, such as mental experiences might be achievable by non-organic means [37]. Indeed, in our view, qualia or phenomenal states can be described in terms of emergent processes of information, which can have downward causation, but arise only when the information is physically embodied.

This view is comparable to Chalmers' 'double-aspect theory of information'. Chalmers also holds that "we might say that phenomenal properties are the internal aspect of information. The informational view allows us to understand how experience might have a subtle kind of causal relevance in virtue of its status as the intrinsic aspect of the physical” [2]. The view is also compatible with Davidson's anomalous monism, rebutting Kim's epiphenomenalism and Edelman's pessimism on the causal nature of qualia. 
Though qualia and consciousness can be characterized in terms of information, they can only be characterized as such by considering them as "embodied information". This is a difference that makes a difference. It also follows that a purely functional description of mental states and consciousness is impossible since pure functionalism holds that mental phenomena can be completely characterized as instantiations of machine operations. Furthermore, the emergence of mental states could, in principle, be described by the theory of nonlinear complex dynamical systems, though a lot of empirical research still seems necessary [29]. Therefore, Chalmers' remarks on the use of nonlinear and chaotic dynamics seem to be unjustified [2]. One of the reasons we think this is the case is that the framework of complex nonlinear dynamical systems was never meant to explain the individual experiences of the qualia, "the what-is-it-like” phenomenal side of the qualia, but, rather, their emergence and dynamics [6]. However, this does not mean that we cannot go one step further and indicate how emergent phenomenal states may lead to a conscious experience. In fact, the concepts of emergence and downward causation introduced here also have implications for the empirical study of consciousness and are, therefore, more than just a conceptual framework. These concepts are becoming more widely recognized in empirical consciousness studies. Recent proposals by E. Thompson and F. Varela can be used to illustrate this [41]. In a framework describing the relationships between neural dynamics, consciousness, and embodiment, they propose to investigate the neural correlates of consciousness. In doing so, they do not assume only a one-way (upward) causal relationship between neural systems and the contents of consciousness, but also the possibility of a two-way (downward) causal relationship between embodied conscious states and neuronal activity [41]. Using the tools of dynamical systems theory, they suggest that as a result of emergence in complex systems, it is to be expected that in such systems, both upward causation and downward causation are implied in the relationship between neural activity and conscious activity. Since specific cognitive acts demand the integration of distributed and interacting areas of the brain, the search for a neural correlate of consciousness must account for these large-scale integrations of brain activity.

The mechanism they suggest for this integration is the formation of dynamic links mediated by the synchronization of neuronal discharges over multiple frequency bands. Such neural processes constitute paradigmatic examples of self-organization and, in their view, exhibit emergent processes. These processes correspond to the collective behaviors of larger units "in which positive and negative feedback interactions gave rise to non-proportional (or nonlinear) consequences" [41] and which are characterized by both upward and downward causation, "whereby low global characteristics of a system govern or constrain local interactions, [which] plays a key role in an important recent analysis that uses dynamical systems theory to understand intentional action” [41]. The reference made here is to Juarrero, whose concept of downward causation was discussed earlier in this paper [34]. The coupled dynamics of brain, body, and environment, then, exhibit self-organization and emergent processes involving both upward and downward causation at multiple levels, including at the level of conscious acts in relation to neural activity. An important consequence of this is that these processes can be studied empirically. Thompson and Varela mention, as examples of this, the study of human epileptic activity and voluntary perceptual reversal. Human epileptic activity freely modifies the subject's mental competencies, but the converse would also seem to be the case. The subject can voluntarily affect his or her electrical condition that normally would lead to an epileptic seizure [41]. Furthermore, models of the visual perception of multi-stable or ambiguous figures suggest that such perception is based on generic properties of coupled 
non-linear oscillators and their phase relationships, which might show "that different 'cognitive' interpretations of ambiguous figures initiated by the subject might shift the neuronal bias that defines the perceptual reversal” [41]. Emergence and downward causation are, therefore, mechanisms that can account for the causal activity of embodied consciousness.

\section{Conclusion: Is There an Extra Ingredient?}

We have seen how qualia and conscious mental states can be causally active entities, whence they emerge, and why the kind of downward causality they exhibit does not contradict the physical closure of the world. The theory presented here is non-reductive and semi-functionalist. It allows for the emergence of mental states that are informational, but not fully functionalizable, since the information is embodied information. What about the "explanatory gap” we started out with in the beginning? On the face of it, and up to a certain extent, this explanatory gap is a consequence of an asymmetry between the third-and first-person perspective: "due to this asymmetry, we have to expect such a "phenomenal difference" even if we observe one and the same process from either perspective, and we can explain why this is so" [42].

Our discussion has tried to make it clear that if we want to construct a scientific theory of consciousness, we will also need an account of qualia and of phenomenal consciousness. This invariably implies a subjective-experiential aspect, which can be called the hard problem of consciousness [2]. Nagel [9] has already put it that if an organism is conscious, there is something it is like to be such a conscious organism. The aim of this paper has been to show that an attempt to account for this experience need not imply that consciousness could not be studied scientifically, even if the theory aims at being a non-reductive one. In such a theory, the embodiment of the mental is a necessary prerequisite. The theory of non-linear complex dynamical systems can, in this view, be an indispensable tool to understanding the dynamics of neuronal activity. Thus, D. Chalmers' pessimistic view in this respect [2] might be unjustified. This is the more so because within non-linear complex dynamical systems, the concepts of emergence and downward causation can find a natural explanation. On the philosophical level, Davidson's anomalous monism can provide a unifying framework intended to show that epiphenomenalism is not a necessary consequence of a non-reductive theory of consciousness and qualia. To the contrary, the theory we have presented does not deny the causal closure of the world, but hinges on the embodiment of the mental. If the mind, therefore, is indeed meat, it is because of this very same fact that it is more than just that.

\section{Acknowledgment}

The author is grateful to the anonymous referees for helpful comments and suggestions.

\section{References}

1. Wittgenstein, L. Philosophical Investigations; Blackwell: Oxford, UK, 1988.

2. Chalmers, D. Facing up the problem of consciousness. J. Conscious. Stud. 1995, 2, 200-219.

3. Macdonald, C. Mind-Body Identity Theories; Routledge: London, UK, 1989.

4. Levine, J. Materialism and qualia: The explanatory gap. Pac. Phil. Quart. 1983, 64, 354-361. 
5. Vergauwen, R. Realism, reference and logic. In Logique en Perspective; Beets, F., Gillet, E., Eds.; Ousia: Brussels, Belgium, 2000; pp. 365-398.

6. Mainzer, K. Thinking in Complexity: The Computational Dynamics of Matter, Mind and Mankind; Springer-Verlag: Heidelberg, Germany, 2007.

7. Globus, G. Quantum Closures and Disclosures; John Benjamins: Amsterdam, The Netherlands, 2003.

8. Kim, J. Philosophy of Mind; Westview Press: Boulder, CO, USA, 1998.

9. Jackson, F. Epiphenomenal qualia. Phil. Quart. 1982, 32, 127-136.

10. Nagel, T. What is it like to be a bat? Phil. Rev. 1972, 82, 435-456.

11. Shoemaker, S. Functionalism and qualia. Phil. Stud. 1975, 27, 291-315.

12. Shoemaker, S. The mind-body problem. In Minds and Bodies; Wilkinson, R., Ed.; Routledge: London, UK, 2000; pp. 193-197.

13. Kim, J. Physicalism, or Something near Enough; Princeton University Press: Princeton, NJ, USA, 2005.

14. Murphey, N.; Brown, W. Did My Neurons Make Me Do It?; Oxford University Press: Oxford, UK, 2007.

15. Dennett, D. Quining qualia. In Consciousness in Contemporary Science; Marcel, A.; Bisiach, E., Eds.; Clarendon Press: Oxford, UK, 1988; pp. 42-77.

16. Pauen, M. Feeling causes. J. Conscious. Stud. 2006, 13, 129-152.

17. Pauen, M. Painless pain: Property dualism and the causal role of phenomenal consciousness. Am. Phil. Quart. 2000, 37, 51-63.

18. Seager, W. Functionalism, qualia and causation. Mind 1983, XCII, 174-188.

19. Davidson, D. Mental events. In Donald Davidson: Essays on Actions and Events; Clarendon Press: Oxford, UK, 1980; pp. 207-227.

20. Antony, M. Davidson’s argument for monism. Synthese 2003, 135, 1-12.

21. Joseph, M. Donald Davidson; Acumen Press: Chesham, UK, 2004.

22. Davidson, D. Subjective, Intersubjective and Objective; Oxford University Press: Oxford, UK, 2001.

23. Walmsley, J. Explanation in dynamical cognitive science. Mind. Mach. 2008, 18, 331-348.

24. Edelman, G. Naturalizing consciousness. A theoretical framework. Proc. Natl. Acad. Sci. 2003, 100, 5520-5524.

25. Edelman, G.; Tononi, G. A Universe of Consciousness: How Matter becomes Imagination; Basic Books: New York, NY, USA, 2000.

26. Edelman, G. The Remembered Present: A Biological Theory of Consciousness; Basic Books: New York, NY, USA, 1989.

27. Vadén, T. Qualifying qualia through the skyhook principle. Inquiry 2001, 44, 149-170.

28. Mainzer, K. Challenges of complexity in the 21st century. An interdisciplinary introduction. Eur. Rev. 2009, 17, 219-236.

29. Mainzer, K. From embodied mind to embodied robotics: Humanities and system theoretical aspects. J. Physiology-Paris 2009, 103, 296-304.

30. Searle, J. Minds, brains and programs. Behav. Brain Sci. 1980, 3, 417-424.

31. Penrose, R. Shadows of the Mind; Oxford University Press: Oxford, UK, 1994. 
32. Mc Ginn, C. Can we solve the mind-body problem? Mind 1989, 98, 349-366.

33. Kim, J. The non-reductionist's troubles with mental causation. In Mental Causation; Heil, J., Mele, A., Eds.; Clarendon Press: Oxford, UK, 1993; pp. 189-210.

34. Juarrero, A. Dynamics in Action; MIT Press: Cambridge, MA, USA, 1999.

35. Van Gulick, R. Who's in charge here? And who's doing all the work? In Consciousness in Contemporary Science; Heil, J., Mele, A., Eds.; Clarendon Press: Oxford, UK, 1988; pp. 233-256.

36. Dempsey, L.; Shani, I. Dynamical agents: Consciousness, causation and two spectres of epiphenomenalism. Phenom. Cogn. Sci. 2009, 8, 225-243.

37. Deacon, T. Three levels of emergent phenomena. In Evolution and Emergence: Systems, Organisms, Persons; Murphy, N., Stoeger, W., Eds.; Oxford University Press: Oxford, UK, 2007; pp. 88-110.

38. Seth, A. Measuring emergence via nonlinear Granger causality. Artif. Life 2008, XI, 545-552.

39. Mainzer, K. Symmetry and complexity in dynamical systems. Eur. Rev. 2009, 13, 29-48.

40. Collier, J. Information originates in symmetry breaking. Symmetry Cult. Sci. 1996, 7, 247-256.

41. Thompson, E.; Varela, F. Radical embodiment: Neural dynamics and consciousness. Trends Cognit. Sci. 2001, 5, 418-425.

42. Pauen, M. Is there an empirical answer to the explanatory gap argument? Conscious.Cogn. 1998, 7 , 202-205.

(C) 2010 by the authors; licensee MDPI, Basel, Switzerland. This article is an Open Access article distributed under the terms and conditions of the Creative Commons Attribution license (http://creativecommons.org/licenses/by/3.0/). 